\title{
Function at the junction: dynamic interactions between lung cells and extracellular matrix
}

\author{
John R Spurzem
}

\begin{abstract}
Recent advances are elucidating the mechanisms by which cells communicate with the surrounding matrix. Cells have specific receptors for matrix proteins. A number of intracellular molecules with signalling functions aggregate at specialised focal adhesion points and facilitate transfer of information both into and out of cells. The importance of these signalling processes to cell biology makes it likely that manipulation of these processes will allow innovative therapeutic approaches to lung disease.

(Thorax 1996;51:956-958)
\end{abstract}

Keywords: integrin, extracellular matrix, lung diseases, signal transduction.

Some years ago a student of pulmonary physiology might have assumed that lung cells and their surrounding matrix served primarily structural and mechanical roles. Recent advances have shown that lung cells interact with extracellular matrix in dynamic relationships. The cell/matrix interaction is a two way street in that cells both influence and derive information from the matrix. ${ }^{12}$ Adhesion receptors such as integrins allow cells to interact specifically with matrix molecules. Cell interactions with matrix proteins are particularly important during processes such as cell migration and proliferation.

The ability of epithelial cells, fibroblasts, and smooth muscle cells to interact with extracellular matrix is probably important in the pathogenesis of respiratory disorders. In asthma there is significant epithelial damage requiring repair of the epithelium. Inadequate repair of ongoing damage has been implicated in bronchial hyperresponsiveness. Similarly, type II alveolar epithelial cells are responsible for repopulating damaged alveoli. During the repair process epithelial cells must attach to a provisional matrix that contains matrix proteins of inflammation (fibronectin, vitronectin, and fibrinogen). The ability of cells to alter their adhesiveness to various matrix proteins would appear to be a critical step in the complex process of cell migration. ${ }^{3}$
Fibrosis in lung disorders can be viewed as disordered repair processes in which fibroblast and matrix accumulation proceeds unchecked. Anchorage to matrix molecules is required for proliferation and migration of fibroblasts and epithelial cells. Matrix production by fibroblasts and epithelial cells is influenced by matrix derived signals. ${ }^{4}$ Thus, matrix and growth factors work cooperatively in controlling mesenchymal and epithelial cell growth and differentiation.

\section{Scientific basis}

It is now known that cells interact with specific matrix elements using an assortment of cell surface molecules. Several types of receptors have been described including, in particular, the family of integrins. Profiles of integrin expression on lung cells have been reported. Integrins are heterodimers of alpha and beta chains, and at least 14 different alpha chains and eight different beta chains have been described. The various possible $\alpha / \beta$ combinations determine the ligand binding specificities. Integrins bind to specific peptide sequences in matrix proteins and can associate together on the cell surface to form assemblies of receptors that have been called focal adhesions. ${ }^{6}$ Focal adhesions are sites where cytoskeletal elements including actin interact with the cytoplasmic tails of the receptors and strengthen the anchorage points. A collection of proteins localises to focal adhesions and facilitates the interactions of actin filaments with integrins. Importantly, proteins with enzymatic activity that are potentially active in signalling mechanisms can be found in focal adhesions (fig 1).

\section{INTRACELLULAR SIGNALLING}

A signalling molecule known as focal adhesion kinase ( $\mathrm{pp} 125^{\mathrm{FAK}}$ ) that is unique to focal adhesions has been described. ${ }^{2}$ Binding of fibronectin to cells can lead to the start of a signalling cascade that begins with phosphorylation (transfer of a phosphate group from ATP) of tyrosine residues in focal adhesion kinase resulting in increased kinase activity (ability to catalyse the phosphorylation of other proteins). ${ }^{2}$ Importantly, signalling via focal adhesion kinase is known to intersect with other 
signalling systems that are related to oncogenes and growth factors. Tyrosine phosphorylation events triggered in fibroblasts by integrins are similar to the phosphorylation events seen after transformation by the oncogene $\mathrm{pp} 60^{\text {src }}$. Two signalling pathways that are important for growth factor mediated signalling are the ras and mitogen activated protein (MAP) cascades. Recent studies directly link integrin mediated signalling through focal adhesion kinase to the ras signal pathway ${ }^{7}$ and to the MAP kinases. ${ }^{8}$ It is apparent then that interactions of matrix components with integrins on the surface of cells transmit information into cells (outside-in signalling).

INSIDE-OUT SIGNALLING

There are also many examples of cells that modify the affinity and specificity of integrins for their extracellular ligands. This direction of information transfer has been termed insideout signalling. ${ }^{1}$ There are examples of inactivation of a fibronectin receptor, $\alpha_{5} \beta_{1}$ integrin, on several cell types. Experiments with pp60 $0^{v-s r c}$ transformed fibroblasts have shown that phosphorylation of the $\beta_{1}$ cytoplasmic domain leads to decreased ligand binding.

\section{Therapeutic potential}

Given these important roles for integrins in cell/matrix interactions, it seems reasonable to expect that patterns of integrin expression would vary in lung diseases. The expression of an integrin associated with epithelial cells, $\alpha_{v} \beta_{6}$, is increased in bronchitic airways, suggesting that modulation of expression of integrins is important in lung injury and repair. ${ }^{9}$

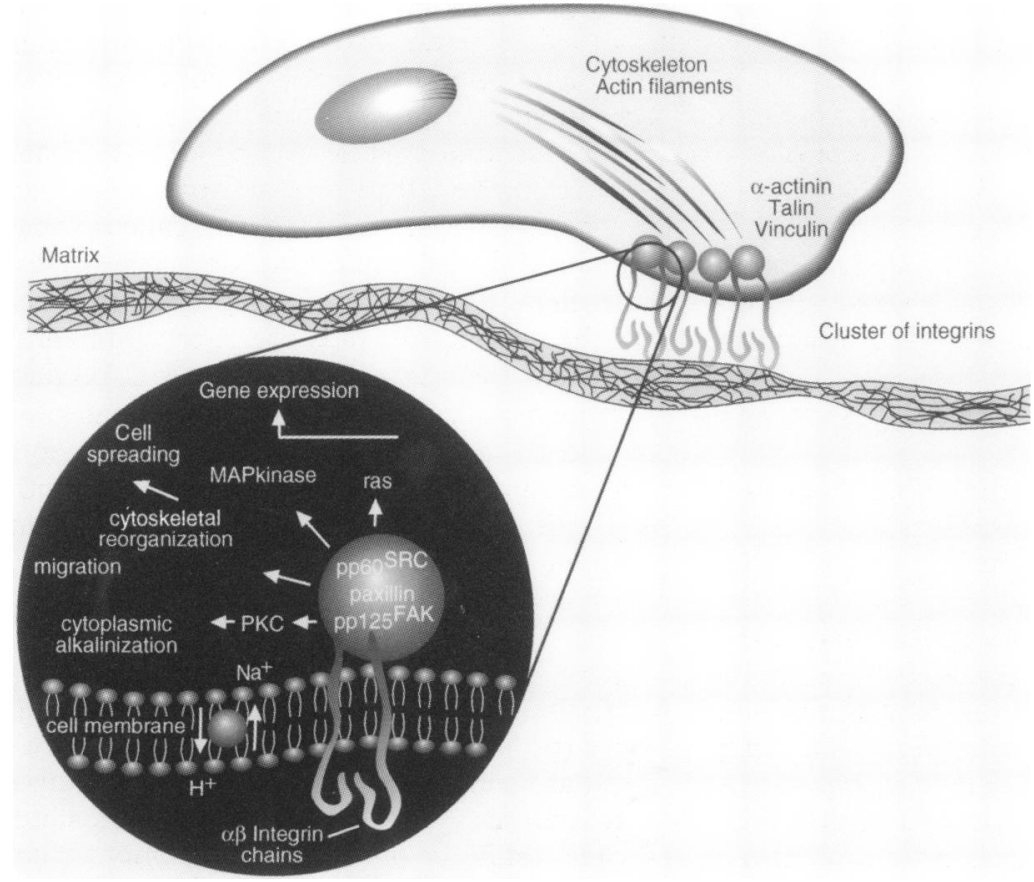

Figure 1 Focal adhesions are sites where cytoskeletal elements interact with a cluster of transmembrane receptors called integrins. It is now known that important molecules, including focal adhesion kinase, with signalling activity are also located in focal adhesions (inset). Signal transduction systems such as ras and MAP kinase, related to oncogenes and growth factors, can be modulated by integrin mediated signalling. The protein kinase $C$ (PKC) family of kinases is also affected, resulting in cytoskeletal reorganisation, cell spreading, and migration.
The complexity of cell/matrix interactions suggests that there are many potential therapeutic interventions. The convergence of growth factor and integrin mediated signalling pathways leads to the idea that modulation of integrin mediated signalling could modulate cellular proliferation and differentiation. Enhanced proliferation and differentiation of epithelial cells might provide an escape from cycles of chronic damage and dysfunction of the epithelium in chronic bronchitis and asthma. Since matrix derived signals are important as regulatory signals for matrix production, alteration of specific integrin function could alter matrix production during fibrotic processes.

The effects of inhibitors of integrin function are being explored in several clinical settings. The idea that blocking integrin function could be clinically useful is supported by teleology. Viper venoms contain platelet integrin inhibitory proteins known as disintegrins. ${ }^{10}$ Based on the knowledge of specific peptide sequences in matrix proteins which bind to integrins, soluble peptides have been designed which block specific integrin function and have been used in vivo. A cyclical peptide which inhibits function of the $\alpha_{\mathrm{v}} \beta_{3}$ integrin causes regression of tumour metastases in animal models. ${ }^{1}$ Integrin blocking peptides have also been used to inhibit coronary arteriopathy in animal models. $^{12}$

\section{Conclusion}

More than just a scaffold to which cells adhere, extracellular matrix is a complex environment that has dynamic relationships with cells. Understanding these relationships and how information is transmitted back and forth from cells should allow us a greater understanding of repair and fibrosis in the lung. Agents which alter the function of specific matrix receptors are already being developed. We are at the cusp of an exciting era in which new therapeutic agents directed at cell/matrix interactions will permit new approaches to lung disease.

1 Hynes R. Integrins: versatility, modulation, and signaling in cell adhesion. Cell 1992;69:11-25.

2 Juliano R, Haskill S. Signal transduction from the extracellular matrix. ₹ Cell Biol 1993;120:577-85.

3 Spurzem JR, Sacco O, Rickard KA, Rennard SI. Transforming growth factor $-\beta$ increases adhesion but not migration of bovine bronchial epithelial cells to matrix proteins. $\mathscr{f}$ Lab Clin Med 1993;122:92-102.

4 Romberger D, Beckmann J, Claasen L, Ertl R, Rennard S Modulation of fibronectin production of bovine bronchial epithelial cells by transforming growth factor- $\beta$. Am Respir Cell Mol Biol 1992;7:149-55.

5 Pilewski J, Albelda S. Adhesion molecules in the lung. An overview. Am Rev Respir Dis 1993;148:S31-7.

6 Turner C, Burridge K. Transmembrane molecular assemblies in cell-extracellular matrix interactions. Curr Opin blies in cell-extracellular

7 Schlaepfer D, Hanks S, Hunter T, van der Geer P. Integrinmediated signal transduction linked to Ras pathway by GRB2 binding to focal adhesion kinase. Nature 1994 372:786-91.

8 Chen Q, Kinch $M$, Lin T, Burridge K, Juliano R. Integrinmediated cell adhesion activates mitogen-activated protein kinases. F Biol Chem 1994;269:26602-5.

9 Weinacker A, Ferrando R, Elliott M, Hogg J, Balmes J Sheppard D. Distribution of integrins avb6 and $a 9 b 1$ and their known ligands, fibronectin and tenascin, in human airways. Am $\mathcal{F}$ Respir Cell Mol Biol 1995;12:547-57.

10 Handagama P, Bainton D, Jacques Y, Conn M, Lazarus $R$ Shuman $M$. Kistrin, an integrin antagonist, blocks endocytosis of fibrinogen into guinea pig mons, blocks and platelet $\alpha$-granules. $\mathcal{F}$ Clin Invest 1993;91:193-200. 
11 Brooks PC, Montgomery AM, Rosenfeld M, Reisfeld $\mathrm{RA}$, Hu $\mathrm{T}$, Klier $\mathrm{G}$, et al. Integrin alpha $\mathrm{v}$ beta antagonists promote tumor regression by inducing apoptosis of angiogenic blood vessels. Cell 1994;79: 1157-64.
12 Molossi S, Elices M, Arrhenius T, Diaz R, Coulber C, Rabinovitch $M$. Blockade of very late antigen-4 binding to fibronectin with connecting segment-1 peptide reduces accelerated coronary arteriopathy in rabbit cardiac allografts. F Clin Invest 1995;95:2601-10. 